\title{
Collision tumor of kidney: A case of renal cell carcinoma with metastases of prostatic adenocarcinoma
}

\author{
Monika Vyas, Santosh Menon, \\ Sangeeta B. Desai \\ Department of Pathology, \\ Tata Memorial Hospital, Parel, \\ Mumbai, Maharashtra, India \\ Address for correspondence: \\ Dr. Sangeeta B. Desai, \\ Professor and Pathologist, \\ Department of Pathology, \\ $8^{\text {th }}$ Floor, Annexe Building, \\ Tata Memorial Hospital, \\ Parel, Mumbai - 400012 , \\ Maharashtra, India. \\ E-mail: \\ sangeetabdesai@rediffmail.com
}

\begin{abstract}
A B S T R A C T
Simultaneous occurrence of prostatic adenocarcinoma and renal cell carcinoma is well documented in the literature. However, metastatic prostatic adenocarcinoma in a kidney harboring a renal cell carcinoma ( $R C C)$ is quite rare. Although renal cell carcinoma is the most common tumor that can harbor metastasis, metastatic prostatic adenocarcinoma in a kidney harboring a RCC is quite rare. There are four cases in the literature showing metastasis of prostatic adenocarcinoma to RCC. However, as per our knowledge, this is the first case of a collision between RCC and metastatic prostatic adenocarcinoma.
\end{abstract}

Key words: Collision tumor, prostatic adenocarcinoma, renal cell carcinoma

\section{INTRODUCTION}

Coexistence of two primary malignancies in the same individual is a well-known phenomenon. However, presence of histologically distinct malignancies in the same organ is quite rare. A true "collision" tumor represents a coexistence of two adjacent but histologically different malignant neoplasms occurring in the same organ without histological admixture or an intermediate cell population zone. ${ }^{[1]}$ Such tumors consist of components with different histogenesis and different tumorigenetic pathways, representing a mosaic of two concurrent but independent tumors that have "collided" with each other. Thus, "collision" tumors are synchronous morphologically different neighboring neoplasms that have expanded into each others' territory and are occurring side-by-side in the same organ. ${ }^{[1]}$

We present an extremely unusual case of a kidney harboring a renal cell carcinoma (RCC) in collision with a metastasis from prostatic adenocarcinoma.

\begin{tabular}{|l|l|}
\hline \multicolumn{3}{|c|}{ Access this article online } \\
\hline Quick Response Code: & Website: \\
\hline & www.jmpo.org \\
\hline
\end{tabular}

\section{CASE REPORT}

A 72-year-old male had complaints of loss of appetite and constipation since 5 months. Per rectal examination revealed an enlarged hard-fixed prostate gland adherent to the rectal wall without luminal compromise. The pre-operative Prostate-Specific Antigen (PSA) was 19.9 ng/ $\mathrm{mL}$. As the patient had locally advanced prostate cancer, he was planned to be managed with neoadjuvant hormonal treatment followed by locoregional pelvic radiotherapy and further hormonal treatment. Hence, he initially underwent a bilateral orchidectomy for hormonal ablation, but defaulted for further treatment. A direct transurethral stenting was performed additionally to relieve him of lower urinary tract symptoms.

The patient presented 8 months later after defaulting, with backache and extensive bone pain. The serum PSA levels and serum alkaline phosphatase were $-950 \mathrm{ng} / \mathrm{mL}$ and $1262 \mathrm{U} / \mathrm{L}$, respectively. The bone scan confirmed extensive skeletal metastasis. On ultrasonography, a left-sided renal mass was noted measuring $5.9 \mathrm{~cm} \times 5.5 \mathrm{~cm} \times 5.4 \mathrm{~cm}$. In addition, multiple enlarged left inguinal and paraaortic nodes were seen. As the mass was solid cystic in nature, radiologic impression of RCC was favored as metastatic prostate cancer was unlikely to be cystic. A left radical nephrectomy was performed at another institute and the specimen was sent as referral for histopathological examination and diagnosis at our tertiary cancer institute. 


\section{Gross pathology}

The radical nephrectomy specimen on gross revealed a well-circumscribed necrotic tumor measuring $5.7 \mathrm{~cm} \times$ $5.2 \mathrm{~cm} \times 4.8 \mathrm{~cm}$ involving the upper two-thirds of the kidney ([Figure 1], thick arrow). The adjacent cortical area appeared firm and pale white ([Figure 1], thin arrow). The renal sinus or the Gerota's fascia was not infiltrated by the tumor. A smooth cyst measuring $4 \mathrm{~cm} \times 3.5 \mathrm{~cm} \times$ $3 \mathrm{~cm}$ was seen at the lower pole ([Figure 1], arrow head). A firm nodule with a grey-white cut-surface measuring $1.2 \mathrm{~cm}$ in diameter was seen adherent to the kidney at the hilum ([Figure 1], asterix). The adrenal gland was unremarkable.

\section{Microscopy}

The tumor at the upper pole was confirmed to be conventional RCC, Fuhrman nuclear grade 2. Random sections from the adjacent pale kidney showed extensive cortical metastasis of prostatic adenocarcinoma, without actual admixture of the two tumor components. The prostatic adenocarcinoma comprised of recognizable acinar/glandular elements in a confluent sheeting pattern. The cyst at the lower pole was a simple cortical cyst. The perinephric fat and the Gerota's fascia were not infiltrated by the tumor. Extensive lymphatic emboli of prostatic adenocarcinoma were noted. The adjacent kidney also revealed features of chronic pyelonephritis. On immunohistochemistry, the tumor at the upper pole (RCC) was positive for CK, vimentin and CD10. The metastatic prostatic adenocarcinoma was negative for CK7, CK20, CD10, HMWCK, vimentin and PSA [Figure 2a-d]. A diagnosis of collision tumor, i.e., conventional RCC along with extensive parenchymal involvement by prostatic adenocarcinoma was rendered. The patient expired 1 month after the surgery due to the extensive nature of the metastatic prostate cancer and debilitating general condition, accentuated by the post-operative status of the patient.

\section{DISCUSSION}

The involvement of the same organ by more than one tumor can be classified on the basis of the manner of involvement by the two tumors:

1. Metastasis of one tumor in the substance of the other, the so-called cancer to cancer metastasis.

2. Presence of two histogenetically distinct tumors in the same organ with histologic admixture of the two tumors, called composite tumors..$^{[1]}$

3. Presence of two histogenetically distinct tumors without histologic admixture of the two entities, called as collision tumors. ${ }^{[1]}$
Documentation of the phenomenon of metastatic carcinoma to a second primary carcinoma must meet certain criteria. These include evidence for the existence of more than one primary tumor, the recipient tumor shown to be a true neoplasm, evidence that the second malignant neoplasm is a true metastasis with established growth or invasion in the host tumor and not due to contiguous growth or embolism of tumor cells. These criteria were established by Campbell and Gilbert. ${ }^{[2]}$ Our case does not meet these criteria as the prostatic adenocarcinoma

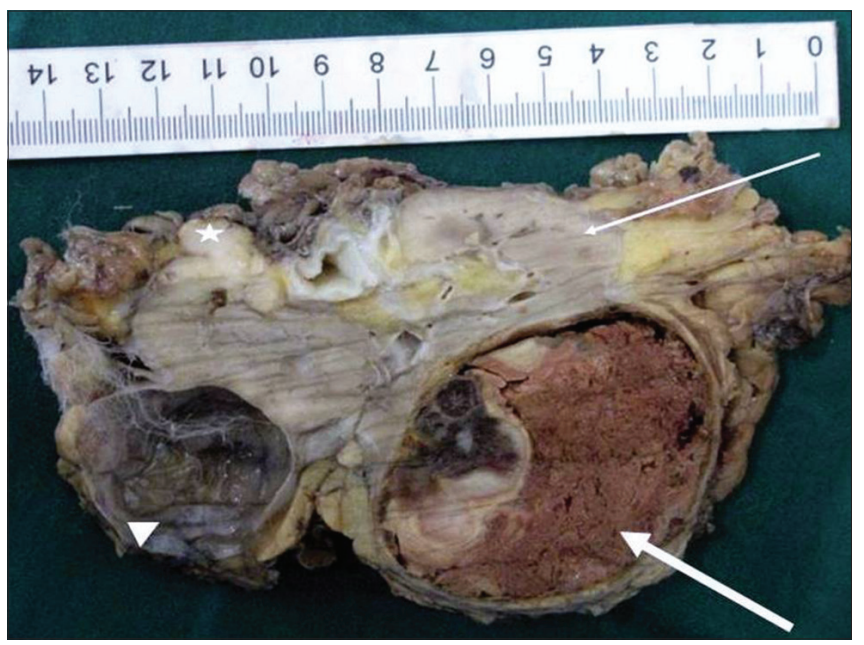

Figure 1: Bisected kidney showing a well-defined mass with extensive necrosis (bold arrow) and a small focus of viable tumor. The adjacent kidney involved diffusely by prostatic carcinoma metastasis appears grossly firm and whitish (thin arrow). Also seen here is a firm whitish irregular deposit (asterix) adjacent to the hilar region. The same kidney also shows a simple cyst (arrow head)

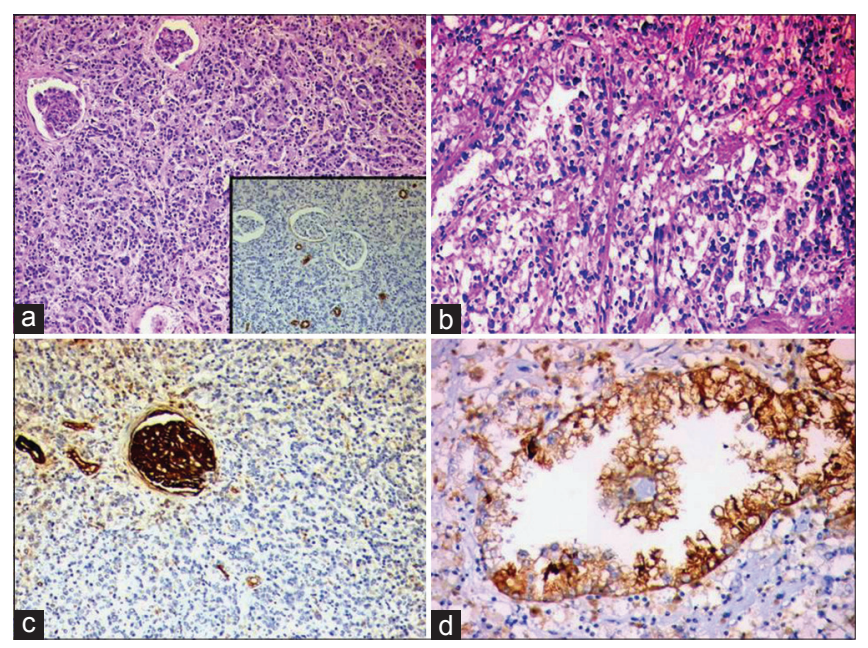

Figure 2: Microscopic and immunohistochemical examination revealed areas with diffuse interstitial infiltration by prostatic adenocarcinoma (a, hematoxylin and eosin, original magnification, $\times 40$ ), which was CK7 negative (a inset, indirect immunoperoxidase) and separate foci of conventional clear cell renal carcinoma (b, hematoxylin and eosin, original magnification, $\times 100$ ). The prostatic adenocarcinoma was negative for CD10 immunomarker (c, indirect immunoperoxidase) in contrast to the renal carcinoma, which was CD10 positive (d, indirect immunoperoxidase) 
does not seed in the RCC. However, it does qualify as a collision tumor, which, by definition, is coexistence of two histologically distinct tumors in the same or contiguous organs without a histologic admixture of the two.

The occurrence of collision tumors is quite rare. The theories relating the occurrence of collision tumors include simultaneous proliferation of two different cell lines, common origin from a pleuripotent precursor stem cell that differentiates into two distinct components, chance opposition of two unrelated tumors ${ }^{[3]}$ and a tumor altering the microenvironment in such a way so as to attract a second primary or metastasis. ${ }^{[1]}$ In this case, chance opposition of the two tumors seems to be the likely explanation. The fact that $25 \%$ of the minute volume passes through the kidney renders the kidneys a likely recipient of metastatic tumors. In addition, RCCs are highly vascular tumors, and are likely to receive a high proportion of the tumor emboli shed by the other tumor. ${ }^{[4]}$ The occurrence of a second primary in patients of RCC is quite common. In one study, the incidence was as high as $26.9 \%$, with $39.4 \%$ being synchronous. The most common second primaries were in the breast, prostate colorectal, bladder and non-Hodgkin lymphoma. ${ }^{[1]}$

RCC has been the most common recipient tumor in tumor to tumor metastasis. ${ }^{[2,4]}$ Four cases of prostatic adenocarcinoma metastasizing to RCC have been documented in the literature. ${ }^{[5]}$ However, we have not come across any case of metastatic prostatic adenocarcinoma in collision with a RCC. The tumors that have been documented to coexist with primary RCC are transitional cell carcinomas, ${ }^{[6]}$ angiomyolipomas, ${ }^{[7]}$ squamous RCCs, ${ }^{[8]}$ collecting duct carcinomas ${ }^{[9]}$ and renal adenocarcinomas and lymphomas. ${ }^{[10]}$ Usually, the renal primary is an incidental finding during the metastatic workup of the other primary, ${ }^{[1]}$ as was true in our case.

Our case also contradicts the view held by others that, in the presence of RCC, the tumor is the favored site of metastases with sparing of the adjacent normal kidney. ${ }^{[4]}$ This hypothesis was based on the fact that RCC, with its high lipid and glycogen content, provides a suitable environment for tumor seeding. ${ }^{[4]}$ In our case, the remainder of the kidney, sparing the RCC, was involved by metastatic prostatic adenocarcinoma. The fact that the phenomenon of "cancer to cancer metastasis" failed to occur in this case was thought-provoking in this context. This suggests that additional factors may be involved in such an unusual phenomenon.

The presence of two tumors in the same may or may not alter the prognosis. The compromise of organ function due to the presence of two tumors may worsen the outcome. A question may arise as to which tumor should be treated first. Ideally, both are to be treated simultaneously but, depending on the clinical situation, the more aggressive cancer is managed first as it is more likely to dictate the outcome and survival. ${ }^{[1]}$

\section{REFERENCES}

1. Rabbani F, Grimaldi G, Russo P. Multiple primary malignancies in renal cell carcinomas. J Urol 1998;160:1255-9.

2. Campbell LV, Gilbert E, Chamberlain CR, Watne AL. Metastasis of cancer to cancer. Cancer 1968;22:635-43.

3. Brahmania M, Kanthan CS, Kanthan R. Collision tumor of the colon - colonic adenocarcinoma and ovarian granulosa cell tumor. World J Surg Oncol 2007;5:118.

4. Sella A, Ro JY. Renal cell cancer: best recipient of tumor-to-tumor metastasis. Urology 1987;30:35-8.

5. Inatomi H, Yamada $\mathrm{Y}$, Okamura T. A case of prostate adenocarcinoma metastasizing to Renal cell carcinoma. Int J Urol 1996;3:155-7.

6. Demir A, Onol FF, Bozkurt S, Turkeri L. Synchronous ipsilateral conventional renal cell and transitional cell carcinoma. Int Urol Nephrol 2004;36:499-502.

7. Jimenez RE, Eble JN, Reuter VE, Epstein JI, Folpe AL, Mariza de Peralta-Venturina, et al. Concurrent Angiomyolipoma and Renal Cell Neoplasia: A Study of 36 Cases. Mod Pathol 2001;14:157-63

8. Fúnez R, Pereda T, Rodrigo I, Robles L, González C. Simultaneous Chromophobe renal cell carcinoma and squamous renal cell carcinoma. Diagn Pathol 2007;2:30

9. Ging Y, Sun X, Haines K, Pins MR. Renal cell carcinoma, chromophobe type, with collecting duct carcinoma and sarcomatoid components. Arch Pathol Lab Med 2003; 127:38-40.

10. Bernie JE, Albers L, Baird S, Parsons CL. Synchronous ipsilateral renal adenocarcinoma, transitional cell carcinoma of renal pelvis and metastatic renal lymphoma.J Urol 2000;164:773-4.

11. Mydlo JH, Agnis JA, Donohoe J, Grob BM. A review of urologic cancer patients with multiple primary malignancies World J Urol 2001;19:240-3.

How to cite this article: Vyas M, Menon S, Desai SB. Collision tumor of kidney: A case of renal cell carcinoma with metastases of prostatic adenocarcinoma. Indian $\mathrm{J}$ Med Paediatr Oncol 2013;34:21-3.

Source of Support: Nil, Conflict of Interest: None declared. 